\title{
Enseñar a litigar en Derecho: una experiencia práctica en la Universidad de Pinar del Río
}

Teaching to litigate in Law: a practical experience at the University of Pinar del Río

Recibido: 6 de octubre de 2019 | Aprobado: 27 de noviembre de 2019

\section{Resumen}

El presente artículo muestra las características y los resultados de un juego de roles llevado a cabo en el área del pregrado universitario en Cuba. El objetivo que se persigue es implementar los juegos de roles como una estrategia ideal para la formación de las competencias profesionales en los estudiantes de Derecho de la Universidad de Pinar del Río. Para lograr dicho objetivo, los estudiantes participaron en la representación del Juicio del Moncada. El diseño metodológico, de corte cualitativo, emplea el método fenomenológico como parte de un estudio de etnografía escolar, que se complementa con la metodología investigación acción-participativa. La recolección de datos se ha realizado a través del análisis de documentos, la observación participante, las encuestas y las entrevistas grupales, con la participación de 22 estudiantes, 6 jueces y 8 profesores de la carrera de Derecho. El aporte teórico radica en la propuesta de un sistema de habilidades litigantes y el aporte práctico consiste en la implementación de los juegos de roles jurídicos, destacándose la representación del Juicio del Moncada como experiencia inicial. Los resultados indican que la propuesta de simulación de juicios orales como método problémico ha tributado significativamente al desarrollo de habilidades litigantes en la educación superior cubana.

Palabras clave: habilidades; juego de roles; litigación; métodos.

* Es Doctora en Ciencias Pedagógicas, Graduada de Licenciatura en Derecho en la Universidad de Pinar del Río, Cuba. Profesora Auxiliar del Departamento de Derecho. Para contactar a la autora: lisett@upr.edu.cu / lisypc87@gmail.com

** Es Licenciada en Derecho, Graduada en la Universidad de Pinar del Río. Labora en la Dirección Provincial de Justicia de Pinar del Río, Cuba. Para contactar a la autora: adoracioncarballomoya@gmail.com

ISSN (en línea): 1814-4152 / Sitio web: http://cuaderno.pucmm.edu.do

Cómo CITAR: Páez, L.D., Carballo, A. (2020). Enseñar a litigar en Derecho: una experiencia práctica en la Universidad de Pinar del Río. Cuaderno de Pedagogía Universitaria, Vol. 17, n. 33 , enero-junio, pp. 17-28 


\section{Abstract}

This article shows the characteristics and results of a role-play conducted in the area of undergraduate studies in Cuba. The objective is to implement role-playing as an ideal strategy for the training of professional skills in the law students of the University of Pinar del Río. To achieve this goal, the students participated in the representation of the Moncada Trial. Methodological, qualitatively cutting design uses the phenomenological method as part of a school ethnography study, which is complemented by the action-participating research methodology. Data collection has been conducted through document analysis, participating observation, surveys and group interviews, with the participation of 22 students, 6 judges and 8 teachers from the law degree. The theoretical contribution lies in the proposal of a system of litigating skills and the practical contribution is the implementation of the legal role-playing, highlighting the representation of the Moncada Trial as initial experience. The results indicate that the proposal to simulate oral trials as a problemic method has significantly contributed to the development of litigating skills in Cuban higher education.

Keywords: skills; role playing; litigation; methods.

\section{Introducción}

Las estrategias encaminadas a lograr una educación de calidad desde una dimensión integradora resultan disímiles, a tono con los Objetivos de Desarrollo Sostenible de la Agenda 2030. El objetivo 16 precisamente está dirigido a promover sociedades pacíficas e inclusivas y facilitar el acceso a la justicia para todos. En tal sentido, los resultados del empleo del juego de roles y las experiencias validadas en la práctica educativa de la carrera de Derecho en Cuba resultan pertinentes y requieren de su visualización y constante perfeccionamiento.

La dinámica de una clase debe trasladarse a otros espacios donde el estudiante se visualice como partícipe del ejercicio profesional. De esta manera, se estaría encontrando con problemas de la práctica, pero con la orientación adecuada, buscará y aplicará una efectiva solución. Este ejercicio simulado de la oratoria le proporcionará las habilidades para perfeccionar el tecnicismo jurídico.

El fin martiano de la educación consistía en preparar al hombre para la vida. Esta educación de carácter científico se propone educar al sujeto desde y para la identidad, con la fusión orgánica de lo instructivo y lo educativo para potenciar la formación en valores y el trabajo. A decir del propio Apóstol y pedagogo cubano: "educar es poner al hombre a nivel de su tiempo, para que flote sobre él” (Martí, 1883, p. 281).

Las universidades cubanas muestran esas instituciones donde se mezcla la teoría de la que emanan los grandes conocimientos con la riqueza de la praxis. Es por eso que, defender una postura activa del estudiante, en la que se enriquezca con conocimientos propios de los que ejercen la carrera de Derecho, no ha de ser solo una pincelada en las llamadas prácticas laborales que se realizan en diferentes órganos como Tribunales y Fiscalías u organizaciones en Cuba, como los denominados Bufetes Colectivos. Se defiende que el período lectivo de un curso escolar debe comprender un equilibrio entre el conocimiento declarativo adquirido a través de la lectura y memorización de leyes y textos jurídicos y la experimentación práctica a través de distintas estrategias como estudios de casos, debates formales e informales, asunción de roles y otras modalidades. Proponemos incluir la asunción de roles, específicamente, como uno de los ítems de la evaluación, como parte del período de prácticas.

El Reglamento de trabajo docente y metodológico del Ministerio de Educación Superior en Cuba (Resolución 2/2018: Artículo 3) define dos pilares básicos del modelo de formación del profesional con perfil amplio. Entre ellos se encuentran la unidad instrucción-educación y el vínculo estudio-trabajo. Este último permite concretar la relación entre el currículo y los modos de actuación del futuro egresado, acercándolo más al ejercicio jurídico en la práctica social.

En este escenario, la implementación de los ejercicios de simulación de juicios orales es una de las formas 
de dar respuestas a problemas educacionales concretos del proceso de formación del jurista, al emplear métodos de enseñanza problémicos que tributen al modo de actuación profesional. Esta experiencia se lleva a cabo en Cuba desde del año 2013 cuando tuvo lugar el Concurso Nacional de Arbitraje Comercial Internacional como propuesta académica con carácter competitivo en el que los estudiantes fungieron como abogados para la resolución de litigios comerciales internacionales mediante el Arbitraje (Páez, 2015).

Las propuestas extracurriculares dirigidas a fomentar las habilidades litigantes del futuro jurista surgen como respuesta a la poca presencia de estrategias de enseñanza que fomenten una participación activa de los estudiantes. Entre los espacios de debate jurídico implementados en el contexto latinoamericano se encuentran los talleres de jurisprudencia, las clínicas, los ejercicios integradores y los debates de juicios simulados (moot courts). Estas tipologías análogas a los juegos de roles han sido desarrolladas en universidades de Argentina, Chile, Colombia, Paraguay y Perú, como alternativas a las carencias pedagógicas y didácticas existentes.

En el caso de Cuba, en particular en la práctica pedagógica de la provincia de Pinar del Río, se han constatado insuficiencias asociadas a la existencia de un diseño curricular más enfocado hacia los conocimientos que hacia las habilidades para litigar; un insuficiente empleo de argumentos jurídicos por parte de los estudiantes para solucionar los problemas de su profesión; escasos conocimientos sobre la lógica procedimental de los actos procesales y un deficiente desarrollo de las habilidades litigantes durante la carrera (Páez, 2015).

Estas deficiencias se detectan debido a que el currículo de la carrera de Derecho en Cuba está diseñado con la previsión de tres tipos de formas de enseñanza que tienen una secuencia a manera de ciclo, a saber: conferencia, clase práctica y seminario, en ese orden. En el caso particular de la Universidad de Pinar del Río, el prototipo de clase práctica que ha predominado es la resolución de casos prácticos orientados por los profesores con antelación, pero que son debatidos con primacía de métodos tradicionales de preguntas y respuestas, por lo que la asunción de roles de verdaderos litigantes continúa siendo una asignatura pendiente.

De este problema descrito se desprende la siguiente pregunta de investigación: ¿Cómo contribuye la implementación de los juegos de roles profesionales en la formación de habilidades litigantes en la carrera de Derecho en la Universidad de Pinar del Río para lograr la integración entre teoría y práctica en el proceso formativo? Esta interrogante tiene en cuenta un proceso asistémico, atomizado y descontextualizado de otras necesidades de la Educación Superior en la provincia más occidental de la isla.

Para darle solución a esta problemática se ha concebido como objetivo: Implementar los juegos de roles como una estrategia ideal para la formación de las competencias profesionales en los estudiantes de Derecho de la Universidad de Pinar del Río, desde el desarrollo de ejercicios profesionales tales como la representación del Juicio del Moncada ${ }^{1}$.

El presente estudio abarca en primera instancia la fundamentación teórica de la investigación, enfatizando en las particularidades de la enseñanza del Derecho y del empleo de los métodos problémicos en esta carrera universitaria. Después, esboza la significación de los juegos de roles en la carrera de Derecho como modelo simbólico de la actividad profesional. A continuación, ofrece una propuesta de tres habilidades litigantes propias del jurista que deben desarrollarse desde los estudios del pregrado. Por último, se presenta la metodología fenomenológica empleada en la investigación, la cual resultó útil para describir y validar la experiencia práctica sobre la representación del juicio del Moncada, simulación que tuvo lugar en

1 Es conocido como tal el juicio incoado por la Causa 37 del año 1953, de los Tribunales de Urgencia de Santiago de Cuba, donde comparecían como acusados los participantes en los asaltos a los cuarteles Moncada y Carlos Manuel de Céspedes, por la comisión del delito "Actos contra los poderes constitucionales del Estado", previsto en el otrora Código de Defensa Social de la República de Cuba, en su artículo 148, con una sanción de privación de libertad de veinte años a muerte. En este acto procesal se destacó la figura de Fidel Castro Ruz, quien participó como acusado y a su vez presentó su alegato de autodefensa "La Historia Me Absolverá", programa político de la Revolución cubana y documento histórico de gran trascendencia para los estudios jurídicos en Cuba. 
la Universidad de Pinar del Río durante el curso escolar 2017-2018.

\section{Marco teórico}

En materia de enseñanza del Derecho es válido destacar que no es esta una enseñanza de igual naturaleza a la propiciada en las carreras pedagógicas. El profesor de Derecho es ante todo un jurista, aunque también es un pedagogo. Es por eso que, ante el título presentado, se hace menester traer a colación algunos conceptos que entremezclen la ciencia pedagógica y la jurídica. Su particular incidencia impacta en lo que se conoce como Didáctica del Derecho, que no es más que la ciencia particular encargada de estudiar el currículo de la ciencia jurídica para perfeccionar su enseñanza.

En tal sentido, la revisión bibliográfica afín al tema de la enseñanza del Derecho describe gráficamente la manera en que se acoge a un estudiante de Derecho en una universidad, quien llega con una visión muy inocente de la vida jurídica, por lo cual debe introducírsele en ella, mediante las clases prácticas, en un estudio más relativista de la profesión para que pueda descubrir los distintos discursos jurídicos y aprender la correcta argumentación, pues en Derecho casi todo es defendible (González-Meneses, citado por Vargas, 2011).

Vargas (2011) considera que los profesores de Derecho ocupan un lugar importante en la formación retórica y la argumentación como habilidades básicas del jurista, o sea, la habilidad técnica de argumentar para persuadir o convencer. Estas expectativas acerca de lo que debe lograr el profesor con el alumno se van estableciendo durante la impartición de la docencia, donde debe fungir la formación de competencias argumentativas como un objetivo primordial. Lo que sucede en la práctica es que la forma de impartir la docencia tiende a utilizar de manera rutinaria la lección magistral. Según Gaete-Quezada (2011), la docencia universitaria continúa con los paradigmas tradicionales, asociados al rol central del profesor en la clase y un rol pasivo del estudiante en su propio proceso de formación, la evaluación se desarrolla generalmente mediante la repetición y memorización de contenidos, y es habitual la preeminencia de la teoría sobre la práctica en los procesos formativos del pregrado universitario, como un aspecto característico dominante. Es por esto que este autor defiende la utilización de estrategias activas aplicadas a los procesos formativos de nivel universitario, lo que resultará de gran importancia para el aprendizaje y para el desarrollo de valores y actitudes, constituyendo las técnicas participativas, tales como el juego de roles o los sociodramas, como eficaces para desarrollar competencias genéricas.

Murillo (2019) plantea como una de las formas de medición a la evaluación del dominio, que es la que se realiza a un individuo para saber si es capaz de aplicar de forma práctica, en el exterior, la teoría aprendida en el aula. Y se ha planteado que, de acuerdo con las teorías constructivistas en la educación, la implicación personal del alumno en su propio aprendizaje contribuye a alimentar su interés en la educación y fomenta su creatividad (Espaliú, 2017). Pero lo frecuente es que el protagonismo en la enseñanza recaiga en el profesor, quien comparte con el estudiante su experticia. Sin embargo, es a través de metodologías activas, tales como los juegos de roles, que se logra este empeño. Esta técnica tan participativa y activa también se inserta dentro de los métodos problémicos, es decir, aquellos que se centran en una enseñanza basada en problemas, la cual constituye un pilar pedagógico, en tanto tributa a la formación de la independencia cognoscitiva y al desarrollo de las competencias de los estudiantes al promover la capacidad autónoma, creadora y el constante auto-mejoramiento del individuo (Vásquez y Caro, citado por Reyes, Carrillo y Caamaño, 2017).

Una categoría esencial para el desarrollo de los juegos de roles en el Derecho resulta la argumentación. El abordaje científico sobre la taxonomía de los argumentos se basa en la Teoría de la Argumentación Jurídica, que tiene su génesis en la clasificación aristotélica de los argumentos dialécticos. Los aportes posteriores de la retórica clásica de Perelman, el diagrama de argumentación de Toulmin y la tópica de Viehweg permiten la tipificación de los argumentos afectivoemotivos (para persuadir) y los argumentos lógicoracionales (para convencer).Perelman enfatiza en la persuasión para la argumentación, en tanto 
Toulmin reinterpreta la lógica formal para ofrecer un modelo de argumentación como proceso dialéctico. (Toulmin, 2007; Páez, 2015).

"La conocida actualmente como teoría de la argumentación jurídica tiene su origen en los años 50, cuando se rechaza la lógica formal para analizar razonamientos jurídicos" (Atienza, 2004, p. 2). En esta etapa las concepciones más relevantes sobre las tipologías de argumentos resultan ser $\mathrm{La}$ tópica de Viehweg, la Nueva retórica de Perelman y la Lógica informal de Toulmin. Se destaca también Edward H. Levi, en 1951, con la obra An introduction to legal reasoning, cuyo esquema básico era el razonamiento mediante ejemplos. Los aportes posteriores corresponden a Robert Alexy, Neil McCormick y Robert Summers, principales representantes de la llamada Teoría estándar de la Argumentación Jurídica (Páez, 2015).

\section{La significación de los juegos de roles en la carrera de derecho}

Álvarez (1999, p. 23) defiende la necesidad de "utilizar el método de la ciencia, como método fundamental de enseñanza y aprendizaje, y de trabajo". En el caso de la ciencia jurídica, la argumentación del razonamiento judicial mediante la litigación aparece como un imperativo insoslayable. Por tanto, para el desarrollo del juicio oral, que es el ejercicio de litigación por antonomasia, se identifica la necesidad del empleo de métodos de enseñanza problémicos, como los juegos de roles, que permiten el desarrollo de habilidades litigantes.

Los métodos de enseñanza problémicos favorecen el desarrollo de la oratoria forense y la argumentación jurídica, en particular, la exposición problémica, el método investigativo y los juegos didácticos creativos. Ellos permiten una mayor independencia del alumno en la construcción del conocimiento, el desarrollo de la oralidad, así como un papel activo y creativo en la asimilación de los contenidos para la solución de problemas de la profesión.

Los métodos de aprendizaje activo revisten especial importancia para la presente investigación. Se destacan tanto los juegos didácticos competitivos (olimpíadas y encuentros de conocimientos) como los profesionales u ocupacionales (análisis de casos, interpretación de papeles, entre otros), de acuerdo con la clasificación brindada por Majmutov (1983). Estos constituyen un modelo simbólico de la actividad profesional y permiten el tránsito de la actividad cognoscitiva a la profesional, de lo que deriva la tendencia a la profesionalización de la enseñanza (Álvarez, 1999).

La poca presencia de estrategias de enseñanzaaprendizaje activo en la carrera de Derecho ha generado la necesidad de emplear métodos problémicos. Y aunque los juegos de roles pudieran parecer una mera representación teatral, a decir de Guzmán (2012, p. 21) "el juicio oral es un ejercicio científico" y, por ende, la formación para la litigación debe desarrollarse como proceso consciente, sistémico y estructurado para el desarrollo de habilidades no histriónicas, sino más bien habilidades litigantes.

\section{La formación de habilidades litigantes en el estudiante de derecho}

A criterio de Álvarez (1999, p. 7), la formación es "el proceso y el resultado cuya función es la de preparar al hombre en todos los aspectos de su personalidad”. Y en la Educación Superior cubana el término formación alude al proceso sustantivo dirigido a preparar integralmente al estudiante universitario, proceso este que incluye tanto los estudios de pregrado como los de postgrado (Horruitiner, 2007). Desde la Didáctica, al estudiar la formación de la habilidad, se entiende esta última "como la dimensión del contenido que muestra el comportamiento del hombre en una rama del saber propia de la cultura de la humanidad. Es, desde el punto de vista psicológico, el sistema de acciones y operaciones dominado por el sujeto que responde a un objetivo" (Álvarez, 1999, p. 71).

La propuesta teórica de las habilidades litigantes se deriva esencialmente del estudio histórico-doctrinal y jurídico de las obras de Baytelman y Duce (2004); Blanco, Rojas, Moreno y Decap, (2005); Moreso (2006); Coscia (2007); Atienza (2008) y Morales (2012). Sus sistematizaciones constituyen bases teóricas para el análisis de las habilidades litigantes desde tres aristas fundamentales: fundamentar posiciones teóricas en debates jurídicos, argumentar jurídicamente la solución legal de un caso y litigar sobre conflictos jurídicos en actos judiciales. 
Las habilidades litigantes, expresadas de manera secuenciada e integrada, se identifican como el sistema de acciones y operaciones que desarrolla el estudiante de Derecho para ejecutar un proceso de litigación. Estas acciones consisten en: elaborar una teoría del caso, practicar las pruebas y defender un alegato frente a un tribunal de justicia.

La elaboración de una teoría del caso implica en primer lugar la identificación de los elementos fáctico, jurídico y probatorio, y posteriormente la redacción del escrito o documento jurídico contentivo de la pretensión judicial. La práctica de pruebas, como acción, se operacionaliza al seleccionar el material probatorio, someterlo a contradicción en juicio, y realizar el interrogatorio a testigos, peritos e intérpretes. Por último, la litigación procesal se dirige a la defensa del alegato, que no es más que la presentación del discurso forense con empleo de la oratoria y la argumentación jurídica. (Páez y Díaz, 2013, p. 446).

El término alegato se emplea de manera particular en las Ciencias Jurídicas. Su etimología, del latín allegātus, significa discurso o exposición donde los argumentos son usados para defender o agredir en determinados contextos. Por lo tanto, el alegato será el instrumento fundamental del litigante, en tanto expresa la concreción de los argumentos de diversa índole que sustentan su teoría del caso.

Para Baytelman y Duce (2004, p.24), "la tarea de litigar puede traducirse en la de proveer al juzgador de un relato verosímil, que le dé fundamento a una decisión en nuestro favor". Para la formación de habilidades litigantes se requiere del empleo de argumentos lógico-racionales y afectivoemotivos. "La argumentación jurídica consiste en la exposición de un discurso oral por medio del cual se pretende justificar determinada decisión judicial en un contexto adversarial" (Salas, 2010, p. 86). Por tanto, la simulación de juicios orales es un escenario propicio para argumentar la tesis del litigante y lograr la emisión de un fallo favorable por parte del juez.

La elocuencia del litigante se demuestra en todo un proceso de distinta naturaleza, ya sea penal, civil, administrativo, laboral u otra rama del Derecho. El hecho es que, las habilidades del litigante se ejercitan en la práctica. Sin dudas, estudiar la historia y acumular elementos que sirvieron de argumentos para sustentar procesos importantes, es una verdadera oda a la memoria y a la justicia.

El juego de roles para la formación de las habilidades litigantes no será una simulación al azar, porque se desvirtuaría la oportunidad de rememorar grandes hitos históricos. Esto significa que, no solo los docentes pueden proponer juicios específicos para su simulación, sino también las instituciones de acogida para las prácticas laborales pueden guiar la simulación del ejercicio profesional mediante una búsqueda en los archivos históricos correspondientes. De esta iniciativa surge el caso en cuestión, que ofrecerá el aporte del juicio del Moncada a la formación del futuro jurista.

En este sentido, debido al impacto que tuvo para América Latina y el mundo "La historia me absolverá", el alegato de autodefensa de Fidel Castro Ruz, líder histórico de la República de Cuba, se ha hecho necesario que los estudiantes universitarios cubanos, especialmente los de la carrera de Derecho, se instruyan en cuanto al valor político y jurídico de este documento y que profundicen el estudio que en sede de la litis originó lo que se convertiría en el programa político de la Revolución cubana. Asimismo, la causa judicial 37 de 1953 tiene un valor pedagógico y didáctico que se materializa con el desempeño de los juegos de roles profesionales, trascendentales para el proceso de enseñanza-aprendizaje del Derecho.

\section{Metodología}

El estudio se ha realizado empleando el método fenomenológico, el cual se basa en la búsqueda de significados a los fenómenos a partir de un análisis racional de las leyes que los rigen (Castillo y Cabrerizo, 2006). En esta ocasión se ha utilizado como técnica propia de dicho método el estudio de los fenómenos pedagógicos en su contexto natural, para captar los significados de la simulación de roles en la formación de habilidades litigantes del futuro jurista.

El método fenomenológico (estudio de casos) se ha empleado como parte de una investigación etnográfica. En particular, se recurrió a la etnografía escolar, empleada para realizar estudios descriptivos de la cultura en la escuela, en este caso de una 
carrera universitaria donde se emplean los juegos de roles. A través de las estrategias utilizadas obtuvimos datos fenomenológicos que permiten representar una concepción del mundo de los propios participantes que a su vez están siendo investigados (Álvarez, 2011).

El ejercicio se llevó a cabo durante el curso 2017 2018, en fecha 25 de noviembre de 2017, al emplear como escenario un tribunal de justicia en Pinar del Río, para contextualizar lo más posible el estudio del caso y comprender su dinámica cultural a través de la simulación en contextos judiciales reales. Para esta investigación etnográfica se empelaron técnicas tales como el análisis de documentos, la entrevista y la observación participante (mediante instrumentos como los diarios de campo y las grabaciones). El juicio oral fue grabado en su totalidad, gracias a lo cual constituye en la actualidad un material de referencia bibliográfico y un medio de enseñanza para el resto del estudiantado de la carrera de Derecho.

El universo o participante en este caso está constituido por 22 estudiantes. A la recolección de datos tributaron 6 jueces y 8 profesores del claustro, cuya intervención permitió ilustrar las dinámicas cotidianas del juego de roles como método problémico para la enseñanza del Derecho. La validación de los datos se realizó mediante la triangulación, teniendo en cuenta la puesta en relación de las aportaciones que realizaron los diferentes agentes implicados en la investigación, incluido el punto de vista del propio investigador, por lo que se empleó la metodología investigación acción participativa (IAP), unificando el rol investigador-docente.

Para la aplicación de los principales resultados, con empleo de la metodología investigación acción, se tuvo en cuenta que las propias investigadoras participaron de la transformación del objeto en el escenario educativo, logrando unidad entre la praxis investigativa y la praxis docente. Precisamente la esencia de esta metodología radica en la unidad entre la práctica y el proceso investigativo, sobre la base de dos ideas cruciales: la decisión del grupo y el compromiso de mejora.

Se seleccionó la metodología de la investigación acción porque es participativa, colaboradora, transformadora, y da paso a un proceso sistemático de aprendizaje, a una rigurosa reflexión de grupo. La misma se estructura en 4 momentos fundamentales: reflexión inicial sobre la situación, planificación, puesta en práctica y observación de cómo funciona, y reflexión (Nocedo, Castellanos, García, Addine, González y Gort, 2001). De forma general, la IAP sigue una espiral de ciclos de planificación, acción, observación, reflexión y luego un nuevo momento para la acción, con nuevas observaciones y reflexiones, las que fueron esenciales para la validación del juego de roles desempeñado.

\section{Una experiencia práctica en la Universidad de Pinar del Río: La representación del juicio del Moncada}

La experiencia de representación de roles litigantes en la universidad pinareña constituyó una práctica experimental para capacitar a futuros egresados. Esta modalidad tuvo lugar por primera vez en la sede que ocupa la Sala Penal del Tribunal Provincial de Pinar del Río. Durante el ejercicio de simulación del Juicio del Moncada los estudiantes fungieron como jueces, abogados y fiscales, radicando la causa 37 de 1953 de los Tribunales de Urgencia de Santiago de Cuba.

Es válido destacar el tributo del ejercicio simulado a la preparación académica del estudiantado en materia de Litigación Penal. Su desarrollo conllevó el análisis de categorías tales como la tipificación del delito de Actos contra los Poderes Constitucionales del Estado (artículo 148 del Código de Defensa Social), la sanción penal, sus circunstancias modificativas, agravantes, atenuantes, y las irregularidades procesales de la causa incoada. Asimismo, permitió un estudio comparado entre el otrora Código de Defensa Social y el actual Código Penal cubano, lo cual requirió del empleo de los métodos exegético-analítico y jurídico-comparado.

Se resalta además la formación de los discentes en materia de oratoria forense, pues "no son discursos orales desde la perspectiva de la producción los repetidos memorísticamente o los textos escritos leídos, como las noticias de radio o televisión" (Abascal, 1993, p. 21). Por tanto, se requirió que los estudiantes fuesen capaces de demostrar, con empleo de la oratoria forense, el desarrollo de las habilidades litigantes: elaborar una teoría del caso, emplear los argumentos jurídicos y presentar 
un alegato. El objeto del debate lo constituyó precisamente el alegato "La Historia me absolverá", que constituye la pieza oratoria más trascendental del siglo XX cubano.

El estudiante que fungió como fiscal, al abordar los hechos suscitados en los asaltos a los cuarteles Moncada y Carlos Manuel de Céspedes, estructuró su teoría del caso alegando culpabilidad y emitió su discurso sobre la base de catalogar las palabras de Fidel como una "arenga política improcedente". Por otra parte, el litigante que representó a Fidel Castro empleó argumentos de tipo lógico-racionales y afectivo-emotivos (Nuñez y Páez, 2018). Entre ellos se encuentran: "Un principio justo desde el fondo de una cueva puede más que un ejército" (argumento de autoridad, citando a José Martî), "Ustedes sabrán cuál es su deber; yo sé bien cuál es el mío" (argumento lógico-racional), Cuba, iqué sería de ti si hubieras dejado morir a tu Apóstol! (argumento afectivo-emotivo), entre otros; destacándose el texto épico: "Condenadme, no importa, La historia me absolverá".

En ese extraordinario discurso estaba contenido el programa de la Revolución, por lo que el estudio del documento se revierte en competencias jurídicas para el estudiantado. La preparación se evidenció no solo en la elocuencia de Fidel al pronunciar su contundente alegato, sino en las declaraciones de los demás testigos, entre ellos, Melba Hernández, Haydée Santamaría, Juan Almeida, Raúl Castro, y el resto de los moncadistas. Los estudiantes hicieron un estudio minucioso de la obra compilada por la periodista Marta Rojas. Este ejercicio acredita la contribución a la formación histórica de los participantes, unido al desarrollo de la argumentación jurídica y del método histórico-lógico para la solución de problemas de la profesión.

El desarrollo del ejercicio jurídico de roles estuvo orientado a tareas más complejas para los estudiantes, que conllevaron a la estructuración de los alegatos a tenor de la normativa vigente en Cuba, establecida por la Fiscalía General de la República. Tal y como establece la Instrucción No. 7/99: Normas generales para el trabajo de control de los procesos penales. La estructura del informe se dirige hacia los siguientes elementos: introducción, análisis del resultado de la práctica de la prueba, trascendencia social del hecho, fundamentación de la calificación jurídica de los hechos, fundamentación de la calificación de la participación y de las circunstancias que se aprecian, valoración general del caso y explicación de la sanción a solicitar.

Los mayores aportes del juicio simulado para la litigación cubana estuvieron presentes en la fase de juicio oral, que al decir del autor Rivero (2014, p.87) "es la fase decisoria o principal del proceso penal", dando lugar a lo que se conoce históricamente como el Juicio del Moncada. Las vivencias del reconocido juicio llegan con tal claridad a la actualidad debido a que fueron plasmadas por Marta Rojas, una joven recién graduada de la Carrera de Periodismo, quien mediante crónicas narra de una manera imperecedera los sucesos ocurridos en el año 1953 (Leyva, 2001).

La participación de los estudiantes fue de forma selectiva, con roles protagónicos de estudiantes de los años superiores (tercero, cuarto y quinto). No obstante, también participaron estudiantes de segundo año como testigos, sin desempeño como litigantes, y de los primeros años de la carrera como observadores. La representación del juicio fue evaluada por los propios estudiantes como necesaria para su formación profesional, pues constituye uno de los principales acercamientos a la actividad profesional que tiene lugar en los tribunales de justicia. Incluso, los participantes se sintieron identificados con las esferas de actuación profesional que prefiriesen en un futuro para ejercer el Derecho como litigantes.

\section{Resultados}

Para la recogida de la información en esta investigación acción se emplearon métodos como el análisis de documentos y las entrevistas grupales. El primero de ellos posibilitó obtener datos valiosos acerca de los problemas jurídico-penales investigados, pues de la revisión de la causa 37 se pudo constatar la estructuración de cada teoría del caso de las partes litigantes. De igual forma, el análisis de la sentencia permitió la valoración de la argumentación jurídica que desarrollaron los jueces mediante sus resultandos probados.

Las entrevistas realizadas durante el desarrollo de la investigación acción permitieron establecer información directa de las personas (estudiantes, 
profesores y tutores-juristas litigantes), tanto de sus respuestas verbales como no verbales, aprovechando las entrevistas planificadas y también las no planificadas, entendidas estas últimas como charlas informales en determinada coyuntura propicia a la investigación.

La metodología investigación acción participativa (IAP) resultó útil para corroborar los resultados de aplicación de la propuesta, en la medida en que coincidía el profesor como el propio investigador reflexivo de su praxis. De tal forma se logró desarrollar el ejercicio de simulación del Juicio del Moncada. Del mismo resultó que de los 8 docentes participantes estimaron de alta (25\%) y muy alta (75\%) la contribución del juicio oral a la formación profesional de litigantes. En sentido general, fue valorada positivamente la experiencia, la cual generó a su vez nuevas iniciativas para el desarrollo de habilidades litigantes en el estudiantado a través de la propuesta de otros juegos de roles.

En cuanto al nivel de pertinencia de esta actividad extracurricular para desarrollar las habilidades litigantes, los 8 docentes y los 6 jueces entrevistados, así como los 22 estudiantes encuestados, consideran que resultó muy útil el empleo de este juego de roles como método de enseñanza problémica. Su concreción deviene resultado de la impartición de la asignatura Litigación, la cual se enseña en el quinto año de la carrera a estudiantes que están próximos al ejercicio de la judicatura y mediante el desempeño del rol de litigantes se acercan a su modo de actuación profesional.

La formación de habilidades litigantes se evidenció en tanto los estudiantes lograron elaborar un proyecto de informe, seleccionar los argumentos jurídicos y defender oralmente un alegato durante el ejercicio simulado. Las encuestas aplicadas a dichos estudiantes arrojaron resultados positivos, pues un $82 \%$ consideró que la contribución fue muy alta para lograr practicar el interrogatorio y presentar los alegatos, a la vez que contribuyeron a la formación de habilidades litigantes de forma alta (18\%). Incluso, una diferencia sustancial con respecto a los inicios de la investigación radica en que, después de la estrategia implementada, un 90\% de los estudiantes considera trascendental el papel de la litigación en la formación del jurista y en el desarrollo de destrezas profesionales.
La investigación permitió demostrar cómo la simulación de uno de los juicios penales más influyentes del siglo XX cubano desencadenó diferentes tareas que en el ámbito de la enseñanza universitaria tuvieron un gran provecho para la impartición de la asignatura de Litigación. Si bien no se puede obtener datos del desarrollo del juicio oral de las causas de Fidel Castro específicamente desarrolladas en Pinar del Río, las Actas que prevalecen y la presente investigación coadyuvaron al desarrollo de las habilidades litigantes de los estudiantes de Derecho, con particular utilidad para los estudios comparados.

La implementación del juego de roles en la Universidad pinareña constituyó un referente para el análisis exegético-analítico del alegato "La Historia me absolverá" y para el estudio técnico- jurídico del Juicio del Moncada como acto procesal. De manera general, la experiencia resultó muy útil en la Universidad de Pinar del Río, pues el empleo de juegos de roles como método de enseñanza problémico no solo motivó a los estudiantes como actividad extracurricular, sino que impactó en su formación profesional. De igual forma, trascendió a la comunidad pinareña por el impacto cultural y social que implica simular un juicio histórico. La propuesta realizada y ejecutada con la investigación resultó pertinente y a su vez novedosa para el proceso docente educativo en general, respecto al cual se constataron resultados sin precedentes en la práctica pedagógica pinareña.

\section{Conclusión}

El desarrollo de los juegos de roles en la carrera de Derecho ha representado una experiencia sui géneris para la Universidad cubana, en tanto el empleo de este método problémico ha constituido una estrategia ideal para la formación de las competencias profesionales de los estudiantes. La simulación del Juicio del Moncada en la Universidad de Pinar del Río ha tributado significativamente al desarrollo de habilidades litigantes tales como: fundamentar posiciones teóricas, argumentar una solución legal y litigar sobre conflictos jurídicos en actos judiciales.

A la par de los juegos de roles judiciales, las propuestas didácticas de otras universidades latinoamericanas se han enfocado en la realización de talleres de jurisprudencia, clínicas, ejercicios 
integradores y los debates de juicios simulados o moot courts. Su implementación constituye un referente válido para el contexto cubano, toda vez que diversifica las propuestas pedagógicas para la formación de habilidades litigantes. Estas constituyen alternativas a emplear en futuras prácticas con los estudiantes cubanos, asumiendo los retos de intercambiar roles litigantes entre los estudiantes y de simular casos reales de la actualidad judicial, como principales recomendaciones de la investigación.

La práctica experimental llevada a cabo ha permitido reflexionar sobre la necesidad de un replanteamiento didáctico en la enseñanza del Derecho. Esta iniciativa constituye una motivación para que otros contextos puedan replicar la implementación realizada, de manera que contrasten resultados y realicen aportaciones pedagógicas en torno al empleo del juego de roles no solo en escenarios jurídicos, sino también en otras carreras universitarias. Su concreción deberá basarse en sólidos fundamentos científicos, algunos de los cuales han sido esbozados aquí, y otros requieren ser revisados, a modo de prospectiva para futuras investigaciones.

\section{Referencias bibliográficas}

Abascal, M. (2004). La teoría de la oralidad. Andalucía, España: Universidad de Málaga.

Álvarez, C. M. (1999). Didáctica. La escuela en la vida. Tercera edición. La Habana: Editorial Pueblo y Educación.

Álvarez, C. (2011). El interés de la etnografía escolar en la investigación educativa. Estudios Pedagógicos, XXXVII, nº 2: 267-279.

Atienza, M. (2004). Las razones del Derecho. Teorías de la Argumentación Jurídica. Segunda Edición. México: Universidad Nacional Autónoma de México.

Atienza, M. (2008). Constitución y argumentación. La ciencia del Derecho Procesal Constitucional. México: UNAM, IMDPC, Marcial Pons.

Baytelman, A. y Duce, M. (2004). Litigación penal y juicio oral. Chile: Editorial del Fondo de Justicia y Sociedad.
Blanco, R., Rojas, H., A. Moreno, H. y Decap, M. (2005). Litigación estratégica en el nuevo proceso penal, 1ra Edición. Chile: Editorial LexisNexis.

Castillo, S. y Cabrerizo, J. (2006). Formación del profesorado en Educación Superior. Desarrollo curricular y Evaluación. Volumen II. España: McGraw-Hill

Coscia, O. (2007). Litigación y justicia participativa. Cuadernos de Derecho Penal. Extraído el 25 de septiembre de 2012 desde: https:// pensamientopenal.com.ar/15062007/coscia. pdf

Definición y qué es Alegato (2019). Definición y qué es. Disponible en: https://definicionyque. es/alegato/ Recuperado el 24.8.2019.

Espaliú, C. (2017). La aplicación del juego de roles a la enseñanza del derecho de la Unión Europea. Revista de Educación y Derecho Número 15 DOI http://dx.doi.org/10.1344/ REYD2017.15.20947

Gaete-Quezada, R. A. (2011). El juego de roles como estrategia de evaluación de aprendizajes universitarios. Disponible en: https://educacionyeducadores.unisabana edu.co/index.php/eye/article/view/1923/2512

Guzmán, L. E. (2012). El Fiscal ante el juicio oral. Legalidad Socialista, No. 34/2012. Fiscalía General de la República de Cuba.

Horruitiner, P. (2007). La Universidad cubana: el modelo de formación. Capítulo II. El proceso de formación. Sus características. Pedagogía Universitaria, Vol. XII, No. 4.

Instrucción No. 7/99. Normas generales para el trabajo de control de los procesos penales. Fiscalía General de la República (Cuba).

Leyva, J. (2001). Juicio sin garantías y carnicería humana. Disponible en: https://www.bohemia. cu.

Majmutov, M. I. (1983). La enseñanza problémica. La Habana: Editorial Pueblo y Educación.

Martí, J.J. (1883). La América. NewYork, Tomo 8. 
Morales, E. (2012). Litigación oral en el nuevo proceso penal y laboral. Revista de Actualidad Jurídica La Tribuna del Abogado

Moreso, J. J. (2006). Lógica, argumentación e interpretación en el derecho. Barcelona: UOC.

Murillo-Garnica, J. (2019). Repensar la evaluación formativa como parte de los procesos de aprendizaje. RECIE. 3(1), 82-94. https://doi. org/10.32541/recie.2019.v3i1.pp82-94

Nocedo, I., Castellanos B., García, G., Addine, F., González, C. y Gort, M. (2001). Metodología de la investigación educacional. La Habana: Editorial Pueblo y Educación

Núñez, M. P. y Páez, L. D. (2018). Competencia oral, lenguaje jurídico y teoría de la argumentación. Oralia. Análisis del Discurso Oral. Vol. 21(2).

Páez, L. y Díaz, T. (2013). Procedimiento para la formación de juristas en la jurisdicción contenciosa. Revista Avances. Vol. 15, No.4 octubre - diciembre.

Páez, L. D. (2015). Modelo de formación de estudiantes de Derecho para la litigación. Una estrategia para su implementación en la carrera de Derecho. Tesis defendida en opción al Título de Doctor en Ciencias Pedagógicas. Universidad de Pinar del Río, Cuba.

Reglamento de trabajo docente y metodológico de la Educación Superior cubana (2018). Resolución 2 del Ministerio de Educación Superior, Cuba.

Reyes, C.C., Carrillo, S. E. y Caamaño, R.M. (2017). Métodos problémicos e independencia cognoscitiva. Revista Científico Educacional. Vol. 13, No. 2. abril-junio.

Rivero, D. (2014). Estudios sobre el proceso penal. La Habana, Cuba: Ediciones ONBC ISBN: 978-959-7066-92-7.

Salas, C. I. (2010). Trascendencia de las técnicas de litigación oral en el proceso penal. Apreciaciones a partir de la vigencia del Código Procesal penal de 2004. 1ra edición. Lima: Perú.
Serrano Gómez, A. (1992). La pena de muerte en los Códigos Penales de Cuba. Boletín de la facultad de Derecho, núm. 1. Disponible en: http://e-spacio.uned.es/fez/eserv/bibliuned: BFD-1992-1-C81EDE5E/PDF

Toulmin, St. E. (2007). Los usos de la argumentación. Barcelona: Ediciones Península.

Vargas, C. (2011). Metodologías activas en la enseñanza del Derecho: prueba, ensayo y percepción por parte de los alumnos. Comisionado para el Espacio Europeo de la Universidad de Almería. 


\section{Anexo 1}

Imágenes tomadas durante el desarrollo del juego de roles del Juicio del Moncada, representado por estudiantes de la carrera de Derecho de la Universidad de Pinar del Río en el curso escolar 2017-2018.
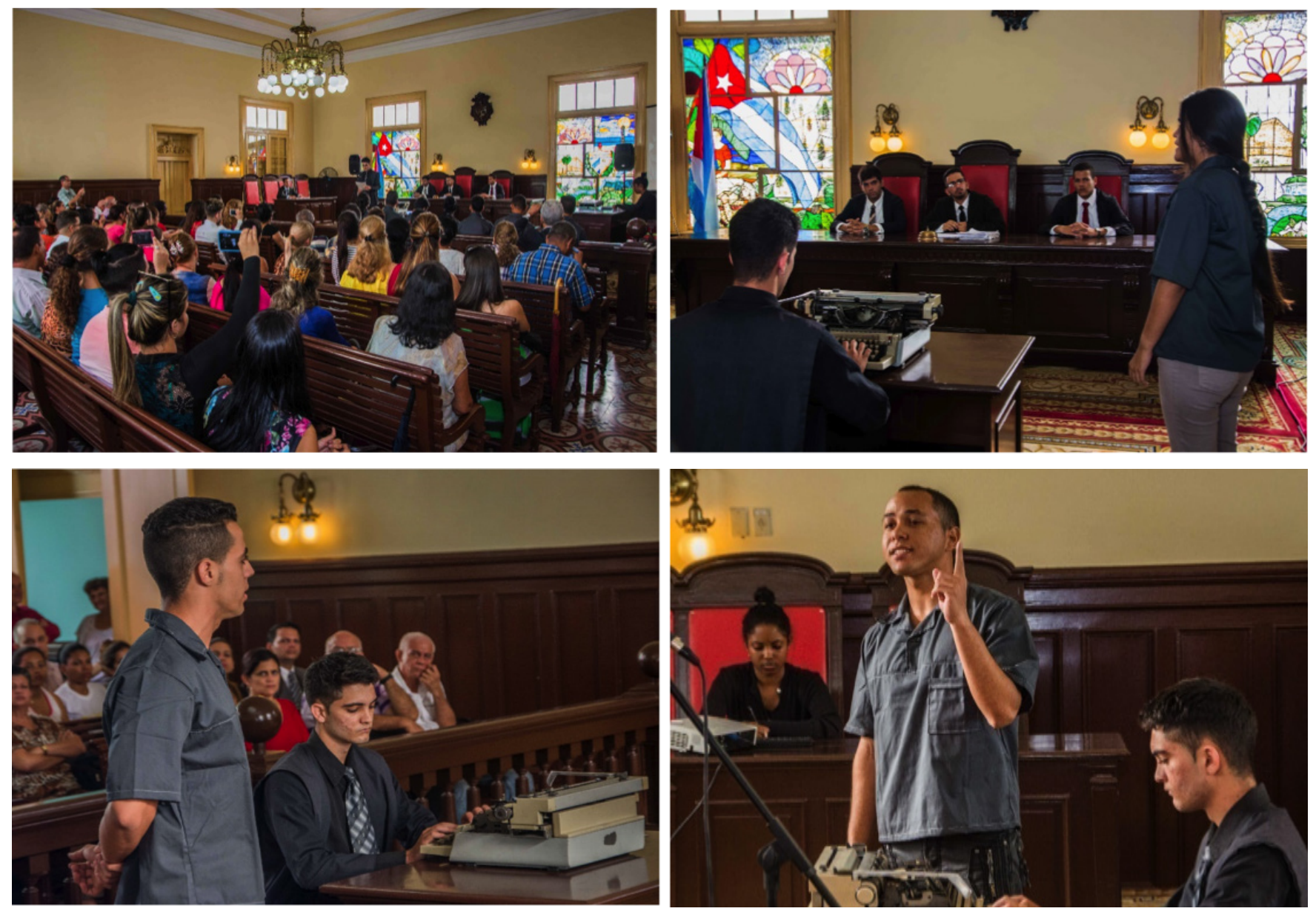